\title{
Drug therapy and other factors associated with the development of acute kidney injury in critically ill patients: a cross-sectional study
}

Danielly Botelho Soares ${ }^{1}$, Juliana Vaz de Melo Mambrini ${ }^{2}$, Gabriela Rebouças Botelho ${ }^{1}$, Flávia Fialho Girundi 1, Fernando Antonio Botoni ${ }^{3}$, Maria Auxiliadora Parreiras Martins ${ }^{\text {Corresp. } 1,3}$

\footnotetext{
1 Faculdade de Farmácia, Universidade Federal de Minas Gerais, Belo Horizonte, MG, Brazil

2 Centro de Pesquisa René Rachou, Fundação Oswaldo Cruz, Belo Horizonte, MG, Brazil

3 Faculdade de Medicina, Universidade Federal de Minas Gerais, Belo Horizonte, Minas Gerais, Brazil

Corresponding Author: Maria Auxiliadora Parreiras Martins

Email address: auxiliadorapmartins@hotmail.com
}

Background: Acute kidney injury (AKI) is associated with a significant increase in morbidity, mortality, and health care costs. In intensive care units (ICU), AKI is commonly multifactorial and frequently involves diverse factors, such as hypovolemia, sepsis, and the use of nephrotoxic drugs. We aimed to investigate drug therapy and other factors associated with the development of AKI in a Brazilian public hospital. Methods: This is a cross-sectional study involving critically ill patients at an ICU of a tertiary hospital. All data on sequential serum creatinine $\left(\mathrm{S}_{\mathrm{Cr}}\right)$ level, glomerular filtration rate (GFR), and urine output were collected during ICU stay. The primary outcome was the occurrence of AKI assessed by the Acute Kidney Injury Network (AKIN) criterion. Sociodemographics, clinical data and drug therapy were considered as covariates. Factors associated with AKI were assessed using logistic regression. Results: Overall, 122 participants were included in the study. Median age was 46.0 (interquartile range, $I Q=29.0-69.0$ ) years, with a predominance of men (58.2\%). Mean number of prescribed drugs throughout ICU stay was $22.0 \pm 9.4$. The number of potentially nephrotoxic drugs ranged from 2-24 per patient. A total of 29 (23.8\%) ICU patients developed AKI. In the AKI-group, patients were older and showed higher Acute Physiology and Chronic Health Evaluation II (APACHE II) score at admission, higher rates of sedation, mechanical ventilation, and infection. More drugs in general and specifically more vasoactive drugs were prescribed for AKI group. Patients who developed AKI tended to have extended stays in the ICU and a lower probability of being discharged alive than patients with no AKI development. Model adjustments of logistic regression showed that the number of medications (OR 1.15; $95 \% \mathrm{Cl} 1.05-1.27$ ) was the only factor associated with AKI in this study. This association was independent of drug nephrotoxicity. Discussion: Intensive care is characterized by its complexity that combines unstable patients, severe diseases, high density of medical interventions, and drug use. We found 
that typical risk factors for AKI showed statistical association on bivariate analysis. The contribution of drug therapy in the occurrence of AKI in medical ICUs reinforces the need for prevention strategies focused on early recognition of renal dysfunction and interventions in drug therapy. These actions would help improve the quality of patient care and ensure progress towards medication safety. 
1 Drug therapy and other factors associated with the development of 2 acute kidney injury in critically ill patients: a cross-sectional study

3

4 Danielly Botelho Soares ${ }^{1}$, Juliana Vaz de Melo Mambrini², Gabriela Rebouças Botelho ${ }^{1}$, Flávia

5 Fialho Girundi ${ }^{1}$, Fernando Antônio Botoni ${ }^{3}$, Maria Auxiliadora Parreiras Martins ${ }^{1,3^{*}}$

7

$8{ }^{1}$ Faculdade de Farmácia, Universidade Federal de Minas Gerais, Belo Horizonte - MG - Brasil;

9 2Centro de Pesquisa René Rachou/Fundação Oswaldo Cruz, Belo Horizonte - MG - Brasil;

${ }^{3}$ Faculdade de Medicina, Universidade Federal de Minas Gerais, Belo Horizonte - MG - Brasil.

*Corresponding author: Professor Maria Auxiliadora Parreiras Martins. E-mail address:

auxiliadorapmartins@hotmail.com

Short title: Factors associated with AKI development 


\section{Abstract}

18 Background: Acute kidney injury ( $\mathrm{AKI})$ is associated with a significant increase in morbidity, mortality, and health care costs. In intensive care units (ICU), AKI is commonly multifactorial and frequently involves diverse factors, such as hypovolemia, sepsis, and the use of nephrotoxic drugs. We aimed to investigate drug therapy and other factors associated with the development of $\mathrm{AKI}$ in a Brazilian public hospital.

Methods: This is a cross-sectional study involving critically ill patients at an ICU of a tertiary hospital. All data on sequential serum creatinine $\left(\mathrm{S}_{\mathrm{Cr}}\right)$ level, glomerular filtration rate (GFR), and urine output were collected during ICU stay. The primary outcome was the occurrence of AKI assessed by the Acute Kidney Injury Network (AKIN) criterion. Sociodemographics, clinical data and drug therapy were considered as covariates. Factors associated with AKI were assessed using logistic regression.

Results: Overall, 122 participants were included in the study. Median age was 46.0 (interquartile range, IQ=29.0-69.0) years, with a predominance of men (58.2\%). Mean number of prescribed drugs throughout ICU stay was $22.0 \pm 9.4$. The number of potentially nephrotoxic drugs ranged from 2-24 per patient. A total of 29 (23.8\%) ICU patients developed AKI. In the AKI-group, patients were older and showed higher Acute Physiology and Chronic Health Evaluation II (APACHE II) score at admission, higher rates of sedation, mechanical ventilation, and infection. More drugs in general and specifically more vasoactive drugs were prescribed for AKI group. Patients who developed AKI tended to have extended stays in the ICU and a lower probability of being discharged alive than patients with no AKI development. Model adjustments of logistic regression showed that the number of medications (OR 1.15; 95\% $\mathrm{Cl} 1.05-1.27$ ) was the only factor associated with AKI in this study. This association was independent of drug nephrotoxicity. Discussion: Intensive care is characterized by its complexity that combines unstable patients, severe diseases, high density of medical interventions, and drug use. We found that typical risk factors for AKI showed statistical association on bivariate analysis. The contribution of drug therapy in the occurrence of AKI in medical ICUs reinforces the need for prevention strategies focused on early recognition of renal dysfunction and interventions in drug therapy. These actions would help improve the quality of patient care and ensure progress towards medication safety. 


\section{INTRODUCTION}

Acute kidney injury (AKI) refers to a sudden decline in renal function, often secondary to an injury that leads to structural or functional changes in the kidneys (Mehta et al., 2007). It is commonly associated with increased cost and extended hospital stay due to the increased morbidity and mortality associated with it (Chertow et al., 2005; Wang et al., 2012). AKI was reported to occur in $7-22.7 \%$ of hospitalized patients (Wang et al., 2012; Jones \& Devonald, 2013; Sawhney \& Simon; 2017) and this range may extend to 19-67\% among critically ill patients (Mataloun et al., 2006; Herrera-Gutiérrez et al., 2013; Jones \& Devonald, 2013). In intensive care units (ICU), AKI is commonly multifactorial and frequently involves diverse factors such as hypovolemia, sepsis, drugs, hemodynamic disturbances, renal hypoperfusion, intrinsic kidney damage, and post-renal obstruction (Taber \& Mueller, 2006; Pannu \& Nadim, 2008; Dennen, Douglas \& Anderson, 2010).

The conceptual models and definitions for AKI and chronic kidney disease (CKD) have many similarities. Both conditions are characterized primarily by decreased kidney function, which can lead to kidney failure and death, as well as fatal and nonfatal complications in other organ systems. However, the time of onset and duration of functional abnormality is important to distinguish AKI from CKD (Chawla et al., 2017). The Kidney Disease: Improve Global Outcomes (KDIGO) has defined AKI as an abrupt decrease in kidney function that occurs over a period of seven days or less, and CKD as abnormalities in kidney structure or function that persist for $>90$ days (KDIGO group$A K I, 2012)$. These definitions are useful for routine employment for the diagnosis of these conditions in clinical practice and research (Chawla et al., 2017). Patient care should adopt procedures to minimize drug-induced harm related to the use of nephrotoxic drugs, and provide supportive care (Pozzoli, Simonini \& Manunta, 2018).

The complexity of conditions affecting critically ill patients often requires the use of a large variety of drugs. Many of them, individually or in combination, may induce renal injury, but it is difficult to identify an isolated cause for AKI. The global contribution of drug-induced renal dysfunction in medical ICUs remains unclear (Perazella, 2012; Finlay et al., 2013). The use of potentially nephrotoxic drugs may compromise patient safety by inducing adverse events involving renal function. In critically ill patients, 
81 nephrotoxic drugs were reported to contribute to $14-21 \%$ of cases of AKI (Bernieh et al.,

82 2004; Mehta et al., 2004; Uchino et al., 2005; KDIGO-AKI group, 2012). KDIGO

83 guidelines have stated that rates may range from 20-30\% (KDIGO-AKI group, 2012).

84 Proposed mechanisms of $\mathrm{AKI}$ include direct cell toxicity or a reduction in the renal 85 perfusion, and the severity also depends on patient's clinical conditions (Mehta et al., 2004). Regarding all these aspects, in the recent years significant efforts have been

87 made in the development of new tools, predictive models and biomarkers of AKI 88 (Pozzoli, Simonini \& Manunta, 2018).

The role of drug therapy in the development of kidney damage is relevant, but often ignored as a preventable cause of AKI and has not been clearly explored in previous studies thus far (Falconnier et al., 2001; Perazella, 2012; Finlay et al., 2013). Temporal and regional assessments of AKI incidence may be influenced by changes in AKI awareness, recognition and clinical practice (Sawhney \& Simon; 2017). Risk factors associated with AKI in ICU patients is an important topic that deserves further investigation. Thus, this study was designed to examine drug therapy and other factors associated with the development of AKI in critically ill patients at a tertiary public 97 hospital in Brazil. 
100

101

102

103

104

105

106

107

108

109

110

111

112

113

114

115

116

117

118

119

120

121

122

123

124

125

126

127

128

129

130

\section{MATERIAL \& METHODS}

\section{Setting and design}

This is a cross-sectional study involving patients hospitalized in a 35-bed medical ICU of a 312-bed tertiary teaching hospital in Belo Horizonte, Southeast Brazil. This is a referral public hospital focused on clinical and surgical emergency assistance. Standard operating procedures and evidence-based protocols are adopted to provide patient care. Patients admitted to ICU were recruited consecutively from October $1^{\text {st }} 2014$ to February $27^{\text {th }} 2015$. The study was approved by the Institutional Review Board on Research Ethics (approval code CAAE 25655014.1.0000.5149) of Universidade Federal de Minas Gerais. We obtained a signed consent from conscious patients or from relatives of those who were sedated and intubated. Patients' relatives who provided informed consent were also their legally authorized representatives. The primary outcome of this study was the incidence of AKI in the ICU patients, and sociodemographics, clinical data and drug therapy information were considered as covariates.

\section{Study population}

The inclusion criteria were: age $\geq 18$ years, no records of kidney disease, more than 24 hours since admission to ICU, and total hospitalization time before admission to ICU not higher than seven days. To assess previous kidney disease, we considered records of CKD in medical charts, chronic dialysis or significant increase in serum creatinine $\left(\mathrm{S}_{\mathrm{Cr}}\right)$ after hospital admission ( $\geq 50 \%$ ). The reason to include patients with a short hospital stay was to assess patients who had been exposed to less hospital interventions that could affect renal function and serve as nonmeasured confounding factors in the study analyses. For readmissions, only data for the first admission were considered in this study. Patients were excluded if they have records of kidney disease, AKI on admission to ICU, and renal replacement therapy (RRT) prior to hospitalization or ICU admission.

\section{Data collection}

All patients admitted to the ICU during the study period were assessed for potential eligibility on the second day of admission. Data were collected until ICU discharge. 
131 Electronic medical charts were reviewed using a pre-tested questionnaire to collect

132 sociodemographic, clinical, and laboratory data. Attending ICU physicians were blinded

133 to detailed information on the study aims and to the list of patients who had been

134 recruited to participate in this investigation. Patients were characterized by sex, age,

135 race, history of alcohol consumption, and smoking status. Clinical information covered

136 the original hospital department in which the patient was admitted, cause of admission,

137 comorbidities, presence of trauma, sedation, mechanical ventilation, infection and/or

138 sepsis when admitted in the ICU, Acute Physiology and Chronic Health Evaluation II

139 (APACHE II) score and length of stay exclusively at ICU. The need of RRT and

140 evolution to discharge or death was also registered.

141 Regarding renal function, all data on sequential $\mathrm{S}_{\mathrm{Cr}}$ level, glomerular filtration rate

142 (GFR), and urine output were collected during ICU stay. The laboratory kit used to

143 measure $\mathrm{S}_{\mathrm{Cr}}$ was VITROS ${ }^{\circledR}$ (Chemistry Products CREA Slides, Ortho-Clinical

144 Diagnostics, Johnson \& Johnson), which is traceable by isotope dilution-liquid

145 chromatography mass spectrometry (ID-LC/MS). The GFR was determined according

146 to the CKD-EPI (Chronic Kidney Disease Epidemiology Collaboration) formula (Levey et

147 al., 2009). The Acute Kidney Injury Network (AKIN) criterion (Mehta et al., 2007) was

148 used to determine the presence of $A K I$, using as baseline the first $S_{C r}$ recorded upon

149 hospital admission. The reason for choosing this first $\mathrm{S}_{\mathrm{Cr}}$ value during hospitalization

150 was due to the lack of access to patients' medical history prior to hospital admission.

151 Patients were classified to be at the worst stage when they reached their highest

152 recorded $\mathrm{S}_{\mathrm{Cr}}$ value during their ICU stay. The first, the highest, and the last $\mathrm{S}_{\mathrm{Cr}}$

153 measurements at ICU were considered in the final analysis. For patients discharged

154 alive, we described the need for additional treatment due to AKI or the status of

155 recovery (partial or total) from AKI. Status of recovery was based on $\mathrm{S}_{\mathrm{Cr}}$ levels: partial

156 recovery was the reduction of $S_{C r}$ levels at discharge, but they were still considered

157 elevated when compared to baseline levels; total recovery consisted on the reduction of

$158 \mathrm{~S}_{\mathrm{Cr}}$ levels to baseline levels detected at hospital admission.

159 The number of medications prescribed per patient during the ICU stay was extracted

160 from the hospital computerized system. Drugs prescribed in the ICU were classified

161 according to the first level of the Anatomical Therapeutic Chemical (ATC) System (ATC, 
162

163

164

165

166

167

168

169

170

171

172

173

174

175

176

177

178

179

180

181

182

183

184

185

186

187

188

189

190

191

192

2013). Vasoactive and potentially nephrotoxic drugs were listed and analyzed as quantitative variables. Vasoactive drugs encompassed dobutamine, dopamine, epinephrine, nitroglycerin, norepinephrine, and sodium nitroprusside. Owing to the heterogeneity of classification of drug-induced nephrotoxicity in information sources (Bicalho et al., 2015), a drug was considered nephrotoxic when cited by at least one out of the four selected drug information sources: Micromedex ${ }^{\circledR}$ (2014), Medscape ${ }^{\circledR}$ (2014), UpToDate ${ }^{\circledR}$ (2014), and the National Therapeutic Formulary (NTF) (FTN, 2010), which is a print publication that is edited by the Brazilian Ministry of Health. The classification of the potential nephrotoxicity of a drug was based on the definition proposed by Finlay et al. (2013). Based on this list, a comprehensive assessment of drug-induced nephrotoxicity and dose adjustment recommendations across drug information sources by drug classes was provided elsewhere (Bicalho et al., 2015). Drugs administered by topical and ophthalmic routes, germicides, inhaled drugs, dialysis solutions, and other non-drug products were excluded from this classification.

\section{Statistical analysis}

Baseline patient characteristics were registered by double entry using EpiData software (ver. 3.1; EpiData Assoc, Odense M, Denmark). All data were analyzed with the STATA software (StataCorp. 2013. Stata Statistical Software: Release 13. College Station, TX: StataCorp LP). Descriptive statistical methods were applied using measurements of central tendency and variability, according to the distribution of variables. The presence or absence of AKI was used to classify patients into two groups that were compared using bivariate analysis. The assumption of normal distribution for the continuous variables was assessed by the Shapiro-Wilk test. Chi-square and Mann-Whitney tests were used in the non-adjusted analyses to examine differences between frequencies and medians, respectively. The linear correlation between the number of drugs prescribed at ICU and APACHE II was tested by the Spearman's coefficient with significance level of $5 \%$.

Factors associated with AKI were identified by model adjustments of logistic regression. Independent variables with $p$ values $<0.25$ in the bivariate analysis were tested in logistic regression models using blocks of variables. The rationale for using $p$ - 
193 value cut-off point of 0.25 is testing variables at some arbitrary level to help selecting

194 candidates for the multivariate analysis that could not be identified as important 195 variables using more conservative levels, such as 0.05 (Bursac et al., 2008). After

196 adjusting the regression model for each block of covariates, the selected variables were

197 considered in the final model of multiple logistic regression (Enter method). The 198 estimated odds ratios (OR) were presented with a 95\% confidence interval $(\mathrm{Cl})$. All $p$ 199 values were 2-tailed ( $\alpha=0.05)$. The variance inflation factor (VIF) was used to identify 200 the presence of multicollinearity (if VIF>10.00). The model was assessed for its 201 goodness-of-fit using the Hosmer-Lemeshow test. The predictive ability of the model 202 was calculated by the estimates for the area under the receiver operating characteristic 203 (ROC) curve. We also performed post-hoc power calculation for sample size. 
205

206

207

208

209

210

211

212

213

214

215

216

217

218

219

220

221

222

223

224

225

226

227

228

229

230

231

232

233

234

\section{RESULTS}

During the study, the 296 patients admitted at the ICU were assessed for eligibility and 122 were included for follow-up. The flowchart for patient selection and follow-up until ICU discharge is presented in Figure 1.

Most patients were men (58.2\%) with ages ranging from 18-88 years (median 46.0 years; IQ (interquartile range) $=29.0-69.0$ ). Surgical department $(46.7 \%)$ and emergency department $(42.6 \%)$ handled most of the hospital admissions. The top two causes of hospital admissions were polytrauma (17.2\%) and cerebrovascular accident (12.3\%). Most patients had at least one comorbidity at admission (58.2\%), represented mainly by diabetes $(19.7 \%)$, cerebrovascular disease $(16.4 \%)$, and chronic pulmonary disease (15.6\%). Twenty six (21.3\%) patients had three or more comorbidities (Table 1).

The number of drugs prescribed per patient throughout the ICU stay varied from 751 (median 20.0; IQ=15.0-27.0). The number of potentially nephrotoxic drugs ranged from 2-24 per patient with a mean of 9.3; SD (standard deviation) $=4.6$. A total of 83 $(68.0 \%)$ patients received at least one vasoactive drug. All drugs in use were classified according to the first level of ATC showing that the main anatomical groups were "Nervous System" (24.3\%), "Alimentary tract and metabolism" (21.0\%) and "Blood and blood forming organs" (18.0\%) (Table 2). According to the list of potentially nephrotoxic drugs, fentanyl was the most frequently used drug (86; 70.5\%). Nephrotoxic antibiotics were represented by vancomycin $(37 ; 30.3 \%)$, ceftriaxone $(31 ; 25.4 \%)$, polymyxin (31; $25.4 \%)$, cefazolin $(15 ; 12.0 \%)$, amikacin $(13 ; 10.7 \%)$ and gentamicin $(9 ; 7.4 \%)$ (Table 3).

Figure 2 depicts the three chosen values of $\mathrm{S}_{\mathrm{Cr}}$ (value at admission, the highest value during the ICU stay and the last $\mathrm{S}_{\mathrm{Cr}}$ ) and the estimated GFR for the groups with or without $\mathrm{AKI}$. In the AKI group, $\mathrm{S}_{\mathrm{Cr}}$ was higher and estimated GFR was lower than the values presented by the non-AKI group.

According to AKIN criterion, 29 (23.8\%) patients developed AKI. From these, ten $(34.5 \%)$ required hemodialysis at some point of their ICU stay and seven $(24.1 \%)$ reached the AKIN 3 stage. Seven out of 11 deaths corresponded to the AKI group. After discharge, seven patients required interventions due to AKI developed during ICU stay 
235 (clinical follow-up or RRT), five had partial recovery, and ten had total recovery from AKI 236 (Figure 1).

237 Sociodemographic and clinical data were compared between both groups by

238

239

240

241

242

243

244

245

246

247

248

249

250

251

252

253

254

255

256

257

258

259

260 bivariate analysis, which is also shown in Table 1. In the AKI-group, patients were older and showed higher APACHE II score at admission, higher rates of sedation, mechanical ventilation, and infection. More drugs in general and specifically more vasoactive drugs were prescribed for AKI group. Additionally, the group of patients who developed AKI tended to have extended stays in the ICU and a lower probability of being discharged alive than the non-AKI group. Our analysis showed a significant correlation between the number of drugs prescribed at ICU and APACHE II (Spearman's coefficient 0.56; $\mathrm{p}<0.001)$.

After adjusting the logistic regression model, the variables maintained in the final model were age, race, number of comorbidities, length of ICU stay and number of prescribed drugs. Only the total number of drugs was significantly associated with AKI (OR 1.15; 95\% Cl 1.05-1.26) (Table 4). We did not identify multicollinearity for these variables indicated by VIF values $<3.00$. The final model fitted well the data (Hosmer-Lemeshow test $=0.9397$ ) and presented a good predictive ability (area under the ROC curve $=0.8102$ ) (Figure 3). Figure 4 shows that increase in number of drugs prescribed increased the probability of the occurrence of AKI, independent of the potential of those drugs to induce nephrotoxicity. Based on literature data (Santos \& Monteiro, 2015), we determined that our statistical power was equal to $71 \%$, with $95 \%$ confidence, to identify the difference in the probability of development of renal damage in 39 non-exposed and 83 exposed to vasoactive drugs. 


\section{DISCUSSION}

262 Our results showed that $23.8 \%$ of studied patients had AKI during ICU stay at a

263 Brazilian teaching hospital. Previous studies have reported wide variations in the

264 incidence of AKI in ICUs by using different diagnostic criteria. Two studies found 265 incidences of $25.5 \%$ (Ponce et al., 2011) and 39.3\% (Nisula et al., 2013) using AKIN

266 classification. Other authors reported an incidence range of $35.7-47.0 \%$ by using the

267 Risk/Injury/Failure/Loss/End-stage (RIFLE) system (Bagshaw et al., 2008; Wahrhaftig,

268 Correa \& de Souza, 2012; Mohamed et al., 2013). In a prospective study including

26929,269 patients hospitalized at 54 ICUs from 23 countries, Uchino et al. (2005) found

2701738 patients $(5.7 \%$; $95 \% \mathrm{CI} 5.5 \%-6.0 \%)$ who had AKI some time during their ICU stay,

271 with a frequency ranging from $1.4-25.9 \%$ of all patients.

272 Bivariate analysis highlighted the relevant risk factors for AKI that have been

273 investigated individually or in combination by other observational studies (Mataloun et

274 al., 2006; Prakash et al., 2006; Taber \& Mueller, 2006; Bagshaw et al., 2008; Perazella,

275 2012; Wahrhaftig, Correa \& de Souza, 2012; Wang et al., 2012; Herrera-Gutiérrez et al.,

276 2013; Mohamed et al., 2013). We found that typical risk factors were associated with

277 AKI in the studied patients, including advanced age, higher APACHE II score at

278 admission to ICU, sedation, mechanical ventilation, and infection. Clinically, patients

279 presenting these characteristics show a high degree of frailty to develop AKI.

280 Previous studies have discussed the contribution of drugs to the occurrence of AKI

281 in critically ill patients. In the prospective study mentioned earlier, drug-induced

282 nephrotoxicity was a contributing factor identified in $19.0 \%$ of AKI patients (Uchino et al.,

283 2005). Bernieh et al. (2004) retrospectively evaluated 81 patients in a tertiary hospital

284 reporting that in 11 patients (14\%), the exposure to nephrotoxic drugs contributed to the

285 occurrence of AKI. In the present study, age, race, number of comorbidities, length of

286 ICU stay, number of drugs, $\mathrm{S}_{\mathrm{Cr}}$ level, GFR, and APACHE II score at hospital admission

287 were the variables included in the final multiple logistic regression model, but only the

288 number of drugs showed significant statistical association with AKI (OR 1.15;95\% Cl

289 1.05-1.27). Interestingly, nephrotoxicity of the drugs did not appear to be a factor

290 associated with AKI. Certain inferences regarding drug-induced AKI in medical ICUs

291 require further clarification in future studies that should provide clearer definitions for the 
292

293

294

295

296

297

298

299

300

301

302

303

304

305

306

307

308

309

310

311

312

313

314

315

316

317

318

319

320

321

322

assessed nephrotoxic drugs and appropriate adjustments for residual confounding variables. We acknowledge that a global understanding of the development of AKI should take into account the influence of individual and concomitant nephrotoxins, their proposed mechanism of nephrotoxicity, dose, drug potency for toxicity and the duration of drug therapy.

Regarding studies developed in Brazil, Reis \& Cassiani (2011) conducted a crosssectional study with 299 medical records of patients admitted for $\geq 5$ days in an ICU. Drug-induced AKI was the most frequent (22; 16.3\%) adverse drug event in the studied hospital. Other study assessed 524 ICU patients to investigate clinical aspects related to acute tubular necrosis classified as ischemic, mixed and nephrotoxic. There was a low incidence (58/524) of isolated nephrotoxic acute tubular necrosis, defined as exposure to nephrotoxins 72 hours preceding the increase of $\mathrm{SC}_{\mathrm{r}}$ (Santos et al., 2006). In our study, the most frequently used nephrotoxic antibiotics were vancomycin (37; $30.3 \%)$, polymyxin $(31 ; 25.4 \%)$ and ceftriaxone $(31 ; 25.4 \%)$. Mostardeiro et al. (2013) analyzed nephrotoxicity rates in 92 solid organ transplant patients who had taken polymyxin, reporting rates of $25 \%, 30 \%$, and $51 \%$ on days 9,16 , and 29 , respectively, after starting polymyxin. An independent association between duration of polymyxin use and $\mathrm{AKI}$ has been reported ( $p=0.037$; OR 1.06; 95\% CI 1.00-1.12). With respect to the combination of nephrotoxins, Soares et al. (2017) investigated the incidence of AKI in 115 ICU patients who used polymyxin B plus vancomycin (Group I) or polymyxin B alone (Group II), showing higher incidence of AKI in Group I than in Group II (62.7\% vs. $28.5 \%, p=0.005)$. Due to the heterogeneity of studies, there are some limitations for comparison among studies. However, we could state that strategies focused on close monitoring of patients taking nephrotoxic drugs alone or in combination would be relevant to minimize the risks for $\mathrm{AKI}$ development. In our study, other important nephrotoxins had a low frequency of use, such as aminoglycosides and contrast media. Individual drugs may have significant pharmacokinetic alterations in critically ill patients, especially for drugs with a small volume of distribution or a high percentage of binding to plasma proteins. Enteral absorption can be reduced owing to the increase in gastric $\mathrm{pH}$, low intestinal motility, drug-food interactions, and intestinal edema.

Depending on drug properties, drug distribution may be altered as a result of changes in 
323 total body water, blood supply, concentration of plasma proteins and binding affinity,

324 tissue permeability, and pH of biological fluids (Schetz et al., 2005; Perazella, 2012).

325 Drug-induced nephrotoxicity may cause additional damage related to hemodynamic

326

327

328

329

330

331

332

333

334

335

336

337

338

339

340

341

342

343

344

345

346

347

348

349

350

351

352

353 alterations and direct injury to tubular cells (Perazella \& Setaro, 2003). Vasoactive drugs were used by $68.0 \%$ of studied patients. Higher doses of these agents are commonly indicated in critically ill patients to help in adjusting blood pressure by vasoconstriction. However, they may also reduce renal blood flow and cause renal hypoxia and acute tubular necrosis with prolonged use (Taber \& Mueller, 2006). Vasoactive drugs did not appear as an independent factor for AKI in our study.

Intensive care is characterized by a complex environment that combines unstable patients, severe diseases, high density of medical interventions, and drug use (Moyen, Camiré \& Stelfox, 2008). Critically ill patients are at high risk for adverse drug events because of the severity of disease, organ dysfunction, and the number, complexity, and duration of medications administered (Manenti et al., 1998; Padilha et al, 2002; Perazella, 2012; Preslaski et al., 2013). The reason for ICU admission might also contribute to clinical complications, such as AKI. In a prospective intervention study including 138 patients in an ICU in Germany, 68 (49\%) patients had renal dysfunction, and $14 \%(110 / 805)$ of prescribed drugs required consideration of renal function. $A$ potential overdose was found in $48 \%$ (53/110) of drugs and this percentage was reduced to $24 \%(p<0.001)$ after the intervention (Bertsche et al., 2009). Inappropriate doses in patients with renal dysfunction may lead to clinical complications, extended hospital stay and avoidable excess costs. Individualized adjustments of drug doses are essential to maximize therapeutic effectiveness and to ensure patient safety (Falconnier et al., 2001). In this context, the expansion of critical care pharmacy services was stated to be relevant to improve patient outcomes and the quality of care. The inclusion of a clinical pharmacist as a member of the multidisciplinary ICU team was reported to protect patients from drug-induced renal impairment (Preslaski et al., 2013).

The literature displays a paucity of high quality evidence from prospective studies investigating the impact of the use of nephrotoxic drugs and the occurrence of $A K I$ in critical care. Investigations on correct dosing of nephrotoxic or other drugs in patients with AKI would be of clinical relevance. Future studies on this topic should also address 
354 the performance of systems for the early detection of drug-induced AKI and the use of

355 more accurate protocols to improve the management of drug regimens and

356 consequently prevent the expansion of renal injury in patients. Serum and urinary

357 biomarkers in monitoring are promising and exhibit prominent advantages, although

358 their use in clinical practice is still limited (Ferguson, Vaidya \& Bonventre, 2008; Peres

359 et al., 2013). The present study provided a better understanding of the occurrence of

$360 \mathrm{AKI}$ in a Brazilian healthcare scenario and highlighted the number of prescribed

361 medications throughout ICU stay as an important risk factor for this complication. This

362 hypothesis needs further investigation in large multicenter studies designed to address 363 drug-induced AKI.

364 Limitations of this study deserve to be mentioned. First, the use of $\mathrm{S}_{\mathrm{Cr}}$ level as a 365 biomarker for estimating renal function and the eventual inconsistencies in diuresis measurements found on hospital records may introduce some imprecision to data collection and to the employment of AKIN criterion. Although $\mathrm{S}_{\mathrm{Cr}}$ levels have been used as the mainstay for the diagnosis of AKI, it does not accurately reflect GFR. Sensitivity and specificity of $S_{C r}$ measurement are questionable due to the lack of a temporal relationship in providing alterations immediately after the establishment of renal injury, a fact that probably delays AKI diagnosis. Thus, the incidence of AKI reported herein may be underestimated. Second, the ideal baseline $S_{C r}$ to assess renal function would be a recent value provided by family doctor. Taking the first $\mathrm{S}_{\mathrm{Cr}}$ on admission - and not a true baseline $S_{C r}$ - could also underestimate the incidence of AKI since many patients may have had an elevation of $\mathrm{S}_{\mathrm{Cr}}$ upon admission and less variation along the hospitalization precluding the identification of AKI under the AKIN classification. Third, the exclusion of patients with CKD could have interfered in the rates of AKI in this study. Fourth, we do not exclude the influence of unmeasured clinical conditions as contributing factors to AKI, such as delirium, hypotension prior to ICU admission and dosing regimen of prior nephrotoxin use (e.g. long-term use of non-steroidal drugs). Fourth, as the highest AKI stage and the number of drugs were measured throughout the entire ICU stay, it was not possible to draw conclusions on the direction of associations which is a limitation inherent to cross-sectional studies. Finally, the constant changes in ICU prescriptions 
384 may have caused an overestimation of the number of medications, since prescribed

385 drugs could have been suspended prior to administration.

386 


\section{CONCLUSIONS}

388 We found that typical risk factors for AKI showed statistical association on bivariate 389 analysis, including advanced age, higher APACHE II score at admission, sedation, 390 mechanical ventilation, and infection. The number of drugs itself was the only factor 391 independently associated with AKI in the critically ill patients under study, independent 392 of drug nephrotoxicity. The contribution of drug therapy in the occurrence of AKI in 393 medical ICUs reinforces the need for prevention strategies focused on early recognition 394 of renal dysfunction and interventions in drug therapy. These actions would help 395 improve the quality of patient care and ensure progress towards medication safety. 


\section{REFERENCES}

Bagshaw SM, George C, Bellomo R, ANZICS Database Management Committe. 2008. A comparison of the RIFLE and AKIN criteria for acute kidney injury in critically ill patients. Nephrology, Dialysis, Transplantation 23:1569-1574. DOI 10.1093/ndt/gfn009.

Bernieh B, Al Hakim M, Boobes Y, Abuchacra S, Dastoor H. 2004. Pattern of acute renal failure in a tertiary hospital in the United Arab Emirates. Transplantation Proceeding 36:1780-1783. DOI 10.1016/j.transproceed.2004.07.015.

Bertsche T, Fleischer M, Pfaff J, Encke J, Czock D, Haefeli WE. 2009. Pro-active provision of drug information as a technique to address overdosing in intensive-care patients with renal insufficiency. European Journal of Clinical Pharmacology 65:823-829. DOI 10.1007/s00228-009-0643-6.

Bicalho MD, Soares DB, Botoni FA, Reis AMM, Martins MAP. 2015. Drug-Induced Nephrotoxicity and Dose Adjustment Recommendations: Agreement Among Four Drug Information Sources. International Journal of Environmental Research and Public Health 12:11227-11240. DOI 10.3390/ijerph120911227.

Bursac Z, Gauss CH, Williams DK, Hosmer DW. 2008. Purposeful selection of variables in logistic regression. Source Code for Biology and Medicine. 3:17. DOI: 10.1186/1751-0473-3-17.

Chawla LS, Bellomo R, Bihorac A, Goldstein SL, Siew ED, Bagshaw SM, Bittleman D, Cruz D, Endre Z, Fitzgerald RL, Forni L, Kane-Gill SL, Hoste E, Koyner J, Liu KD, Macedo E, Mehta R, Murray P, Nadim M, Ostermann M, Palevsky PM, Pannu N, Rosner M, Wald R, Zarbock A, Ronco C, Kellum JÁ on behalf of the Acute Disease Quality Initiative Workgroup 16. 2017. Acute kidney disease and renal recovery: consensus report of the Acute Disease Quality Initiative (ADQI) 16 Workgroup. Nature Reviews. Nephrology 13:241-257. DOI 10.1038/nrneph.2017.2.

Chertow GM, Burdick E, Honour M, Bonventre JV, Bates DW. 2005. Acute kidney injury, mortality, length of stay, and costs in hospitalized patients. Journal of the American Society of Nephrology 16:3365-3370. DOI 10.1681/ASN.2004090740.

Dennen P, Douglas IS, Anderson R. 2010. Acute kidney injury in the intensive care unit: an update and primer for the intensivist. Critical Care Medicine 38:261-275. DOI 10.1097/CCM.0b013e3181bfb0b5.

Falconnier AD, Haefeli WE, Schoenenberger RA, Surber C, Martin-Facklam M. 2001. Drug dosage in patients with renal failure optimized by immediate concurrent feedback. Journal of General Internal Medicine 16:369-375. DOI 10.1046/j.15251497.2001.016006369.x.

Ferguson MA, Vaidya VS, Bonventre JV. 2008. Biomarkers of nephrotoxic acute kidney injury. Toxicology 245:182-193. DOI 10.1016/j.tox.2007.12.024.

Finlay S, Bray B, Lewington AJ, Hunter-Rowe CT, Banerjee A, Atkinson JM, Jones MC. 2013. Identification of risk factors associated with acute kidney injury in patients admitted to acute medical units. Clinical Medicine (London, England) 13:233-238. DOI 10.7861/clinmedicine.13-3-233. 
440

441

442

443

444

445

446

447

448

449

450

451

452

453

454

455

456

457

458

459

460

461

462

463

464

465

466

467

468

469

470

471

472

473

474

475

476

477

478

479

480

Guidelines for ATC classification and DDD assignment 2013. Oslo (2012). Available at: http://www.whocc.no/filearchive/publications/1_2013guidelines.pdf (accessed 4 January 2016).

Herrera-Gutiérrez ME, Seller-Pérez G, Sánchez-Izquierdo-Riera JÁ, MaynarMoliner J, COFRADE investigators group. 2013. Prevalence of acute kidney injury in intensive care units: the "COrte de prevalencia de disFunción RenAl y DEpuración en críticos" point-prevalence multicenter study. Journal of Critical Care 28:687-694. DOI 10.1016/j.jcrc.2013.05.019.

Jones SL, Devonald MA. 2013. How acute kidney injury is investigated and managed in UK intensive care units--a survey of current practice. Nephrology, Dialysis, Transplantation 28:1186-1190. DOI 10.1093/ndt/gft015.

KDIGO-AKI Group. 2012. KDIGO Clinical Practice Guideline for Acute Kidney Injury. Kidney International Supplement 2:19-36. DOI 10.1038/kisup.2012.3.

KDIGO-CNK Group. 2012. KDIGO 2012 Clinical Practice Guideline for the Evaluation and Management of Chronic Kidney Disease. Kidney International Supplement 3:514. DOI 10.1038/kisup.2012.73.

Levey AS, Stevens LA, Schmid CH, Zhang YL, Castro AF 3rd, Feldman HI, Kusek JW, Eggers P, Van Lente F, Greene T, Coresh J, CKD-EPI (Chronic Kidney Disease Epidemiology Collaboration). 2009. A new equation to estimate glomerular filtration rate. Annals of Internal Medicine 150:604-612. DOI 10.7326/0003-4819-150-9-200905050-00006.

Manenti S, Chaves AB, Leopoldino RS, Padilha KG. 1998. [Adverse effects of drugs in intensive care units: analysis of the administration of electrolyte solutions and antibiotics]. Revista da Escola de Enfermagem da USP 32:369-376.

Mataloun SE, Machado FR, Senna AP, Guimarães HP, Amaral JL. 2006. Incidence, risk factors and prognostic factors of acute renal failure in patients admitted to an intensive care unit. Brazilian Journal of Medical and Biological Research 39:13391347.

Medscape. (2014). Available online: http//reference.medscape.com/pharmacists (accessed on 13 March 2014).

Mehta RL, Kellum JA, Shah SV, Molitoris BA, Ronco C, Warnock DG, Levin A, Acute Kidney Injury Network. 2007. Acute Kidney Injury Network: report of an initiative to improve outcomes in acute kidney injury. Critical Care 11:R31. DOI 10.1186/cc5713.

Mehta RL, Pascual MT, Soroko S, Savage BR, Himmelfarb J, Ikizler TA, Paganini EP, Chertow GM, Program to Improve Care in Acute Renal Disease. 2004. Spectrum of acute renal failure in the intensive care unit: the PICARD experience. Kidney International 66:1613-1621. DOI 10.1111/j.1523-1755.2004.00927.x.

Micromedex. DRUG-REAX ${ }^{\circledR}$ System (Electronic Version). (2014). Available online: http://www-micromedexsolutionscom.ez27.periodicos.capes.gov.br/ (accessed 8 March 2014). 
481

482

483

484

485

486

487

488

489

490

491

492

493

494

495

496

497

498

499

500

501

502

503

504

505

506

507

508

509

510

511

512

513

514

515

516

517

518

519

520

521

522

Mohamed H, Mukhtar A, Mostafa S, Wageh S, Eladawy A, Zaghlol A, Hasanin A, Hosni M, Farrag M, Botros M, Farok S, Lotfi A. 2013. Epidemiology of acute kidney injury in surgical intensive care at University Hospital in Egypt. A prospective observational study. Egyptian Journal of Anaesthesia 29:413-417. DOI 10.1016/j.egja.2013.03.003.

Mostardeiro MM, Pereira CA, Marra AR, Pestana JO, Camargo LF. 2013. Nephrotoxicity and efficacy assessment of polymyxin use in 92 transplant patients. Antimicrobial Agents and Chemotherapy 57:1442-6. DOI: 10.1128/AAC.01329-12. Moyen E, Camiré E, Stelfox HT. 2008. Clinical review: medication errors in critical care. Critical Care (London, England) 12:208. DOI 10.1186/cc6813.

National Therapeutic Formulary. [Formulário Terapêutico Nacional-FTN]. (2010) Rename. 1135.

Nisula S, Vaara ST, Kaukonen KM, Reinikainen M, Koivisto SP, Inkinen O, Poukkanen M, Tianinen P, Pettilä V, Korhonen AM, FINNAKI-QOL Study Group. 2013. Six-month survival and quality of life of intensive care patients with acute kidney injury. Critical Care 17:R250. DOI 10.1186/cc13076.

Padilha KG, Kitahara PH, Gonçalves CC, Sanches AL. 2002. [latrogenic events with medications at the intensive care unit: procedures and feelings expressed by nurses]. Revista da Escola de Enfermagem da USP 36:50-57.

Pannu N, Nadim MK. 2008. An overview of drug-induced acute kidney injury. Critical Care Medicine 36:S216-223. DOI 10.1097/CCM.0b013e318168e375.

Perazella MA, Setaro JF. 2003. Renin-angiotensin-aldosterone system: fundamental aspects and clinical implications in renal and cardiovascular disorders. Journal of Nuclear Cardiology 10:184-196. DOI 10.1067/mnc.2003.392.

Perazella MA. 2012. Drug use and nephrotoxicity in the intensive care unit. Kidney International 81:1172-1178. DOI 10.1038/ki.2010.475.

Peres LA, Cunha Júnior AD, Schäfer AJ, Silva AL, Gaspar AD, Scarpari DF, Alves JB, Girelli Neto R, Oliveira TF. 2013. Biomarkers of acute kidney injury. Jornal Brasileiro de Nefrologia 35:229-236. DOI 10.5935/0101-2800.20130036.

Ponce D, Zorzenon CP, Santos NY, Teixeira UA, Balbi AL. 2011. Acute kidney injury in intensive care unit patients: a prospective study on incidence, risk factors and. Revista Brasileira de Terapia Intensiva 23:321-326. DOI 10.1590/S0103507X2011000300010.

Pozzoli S, Simonini M, Manunta P. 2018. Predicting acute kidney injury: current status and future challenges. Journal of Nephrology 31:209-223. DOI 10.1007/s40620017-0416-8

Prakash J, Murthy AS, Vohra R, Rajak M, Mathur SK. 2006. Acute renal failure in the intensive care unit. The Journal of the Association of Physicians of India 54:784788.

Preslaski CR, Lat I, MacLaren R, Poston J. 2013. Pharmacist contributions as members of the multidisciplinary ICU team. Chest 144:1687-1695. DOI 10.1378/chest.12-1615. 
Reis AM, Cassiani SH. 2011. Adverse drug events in an intensive care unit of a university hospital. European Journal of Clinical Pharmacology 67:625-32. DOI: 10.1007/s00228-010-0987-y.

Santos PR, Monteiro DL. 2015. Acute kidney injury in an intensive care unit of a general hospital with emergency room specializing in trauma: an observational prospective study. BMC Nephrology 16:30. DOI 10.1186/s12882-015-0026-4.

Santos WJ, Zanetta DM, Pires AC, Lobo SM, Lima EQ, Burdmann EA. 2006. Patients with ischaemic, mixed and nephrotoxic acute tubular necrosis in the intensive care unit--a homogeneous population? Critical Care 10:R68.

Sawhney S, Fraser SD. 2017. Epidemiology of AKI: Utilizing Large Databases to Determine the Burden of AKI. Advances in Chronic Kidney Disease. 24:194-204. DOI 10.1053/j.ackd.2017.05.001.

Schetz M, Dasta J, Goldstein S, Golper T. 2005. Drug-induced acute kidney injury. Current Opinion in Critical Care 11:555-565. DOI 10.1097/01.ccx.0000184300.68383.95.

Soares DS, Reis ADF, Silva Junior GBD, Leite TT, Parente Filho SLA, Rocha CVO, Daher EF. 2017. Polymyxin-B and vancomycin-associated acute kidney injury in critically ill patients. Pathogens and Global Health 111:137-142. DOI 10.1080/20477724.2017.1309338.

Taber SS, Mueller BA. 2006. Drug-associated renal dysfunction. Critical Care Clinics 22:357-374. DOI 10.1016/j.ccc.2006.02.003.

Uchino S, Kellum JA, Bellomo R, Doig GS, Morimatsu H, Morgera S, Schetz M, Tan I, Bouman C, Macedo E, Gibney N, Tolwani A, Ronco C, Beginning and Ending Supportive Therapy for the Kidney. (BEST Kidney) Investigators. 2005. Acute renal failure in critically ill patients: a multinational, multicenter study. JAMA 294:813-818. DOI 10.1001/jama.294.7.813.

UpToDate. Available online: http://www.UpToDate.com/home/index.html (accessed 24 March 2014).

Wahrhaftig KM, Correia LC, de Souza CA. 2012. [RIFLE Classification: prospective analysis of the association with mortality in critical ill patients]. Jornal Brasileiro de Nefrologia 34:369-377. DOI 10.5935/0101-2800.20120027.

Wang HE, Muntner P, Chertow GM, Warnock DG. 2012. Acute kidney injury and mortality in hospitalized patients. American Journal of Nephrology 35:349-355. DOI $10.1159 / 000337487$. 


\section{Figure Legends}

559 Figure 1. Flowchart for patient selection and follow-up until ICU discharge. Abbreviations: AKI, 560 acute kidney injury.

561

562

563

564

565

566

567

568

569

570

571
Figure 2. $S_{C r}(A)$ and GFR $(B)$ at three different moments of hospitalization according to AKI and non-AKI groups. Abbreviations: $S_{C r}$, serum creatinine; $A K I$, acute kidney injury; GFR, glomerular filtration rate.

Figure 3. ROC curve for the final model.

Figure 4. Probability of occurrence of AKI according to the number of drugs used by the studied ICU patients. Abbreviations: AKI, acute kidney injury; ICU, intensive care unit. 


\section{Table 1 (on next page)}

Bivariate analysis of sociodemographic, clinical, and medication data 
Table 1. Bivariate analysis of sociodemographic, clinical, and medication data in AKI and nonAKI-groups of patients admitted at a teaching hospital ICU.

\begin{tabular}{|c|c|c|c|c|}
\hline Variables & $\begin{array}{c}\text { Total } \\
(\mathrm{n}=122) \\
\end{array}$ & $\begin{array}{l}\text { Non-AKI group } \\
(\mathrm{n}=93)\end{array}$ & $\begin{array}{c}\text { AKI group } \\
(\mathrm{n}=29)\end{array}$ & $p$-value $e^{a, b}$ \\
\hline \multicolumn{5}{|l|}{ Sociodemographic data } \\
\hline Female sex, n (\%) & $51(41.8)$ & $39(41.9)$ & $12(41.4)$ & $0.96^{a}$ \\
\hline Age, median (IQ) & $46.0(29.0-69.0)$ & $41(28.0-63.0)$ & $62.0(46.0-76.0)$ & $0.01^{b}$ \\
\hline \multicolumn{5}{|l|}{ Race, n (\%) } \\
\hline White & $95(77.9)$ & $76(81.7)$ & $19(65.5)$ & $0.07^{a}$ \\
\hline Non-white & $27(22.1)$ & $17(18.3)$ & $10(34.5)$ & \\
\hline History of ethanol use, $\mathrm{n}(\%)$ & $45(37.8)$ & $34(37.8)$ & $11(37.9)$ & $0.99^{a}$ \\
\hline Smoking status, n (\%) & $35(29.4)$ & $26(28.9)$ & $9(31.0)$ & $0.83^{a}$ \\
\hline \multicolumn{5}{|l|}{ Clinical data } \\
\hline $\begin{array}{l}\text { APACHE II Score at admission } \\
\text { ICU, (IQ) } \\
\text { Comorbidities, n (\%) }\end{array}$ & $21.4(15.0-27.0)$ & $20.1(14.0-25.0)$ & $25.6(20.0-31.0)$ & $<0.01^{b}$ \\
\hline 0 & $51(41.8)$ & $44(47.3)$ & $7(24.1)$ & $0.05^{a}$ \\
\hline $1-2$ & $45(36.9)$ & $33(35.5)$ & $12(41.4)$ & \\
\hline$>2$ & $26(21.3)$ & $16(17.2)$ & $10(34.5)$ & \\
\hline Sedation, n (\%) & $67(54.9)$ & $45(48.4)$ & $22(75.9)$ & $0.01^{\mathrm{a}}$ \\
\hline Trauma, n (\%) & $47(38.5)$ & $40(43.0)$ & $7(24.1)$ & $0.07^{a}$ \\
\hline Mechanical ventilation, $\mathrm{n}(\%)$ & $82(67.2)$ & $58(62.4)$ & $24(82.8)$ & $0.04^{a}$ \\
\hline Infection, n (\%) & $49(40.2)$ & $29(31.2)$ & $20(69.0)$ & $<0.001^{a}$ \\
\hline Sepsis, n (\%) & $20(16.4)$ & $12(12.9)$ & $8(27.6)$ & $0.06^{a}$ \\
\hline $\begin{array}{l}\text { Length of ICU stay }{ }^{c} \text { (days), n (\%) } \\
\text { Up to } 5 \\
\text { From } 6 \text { to } 15 \\
\text { More than } 15 \\
\text { ICU Discharge, } \mathrm{n}(\%)\end{array}$ & $\begin{array}{l}56(45.9) \\
36(29.5) \\
30(24.6)\end{array}$ & $\begin{array}{l}50(53.8) \\
23(24.7) \\
20(21.5)\end{array}$ & $\begin{array}{l}6(20.7) \\
13(44.8) \\
10(34.5)\end{array}$ & $0.01^{a}$ \\
\hline Discharge & $111(91.0)$ & $89(95.7)$ & $22(75.9)$ & $<0.01^{a}$ \\
\hline Death & $11(9.0)$ & $4(4.3)$ & $7(24.1)$ & \\
\hline \multicolumn{5}{|l|}{ Medications } \\
\hline \multicolumn{4}{|l|}{$\begin{array}{l}\text { Number of potentially nephrotoxic } \\
\text { drugs, } \mathrm{n}(\%)\end{array}$} & $<0.001^{b}$ \\
\hline $\begin{array}{l}0-6 \\
7-11 \\
>11\end{array}$ & $\begin{array}{l}34(27.9) \\
55(45.1) \\
33(27.1)\end{array}$ & $\begin{array}{l}30(32.3) \\
42(45.2) \\
21(22.6)\end{array}$ & $\begin{array}{l}4(13.8) \\
13(44.8) \\
12(41.4)\end{array}$ & $0.06^{a}$ \\
\hline \multicolumn{5}{|l|}{ Number of vasoactive drugs, $n(\%)$} \\
\hline 0 & $39(32.0)$ & $35(37.6)$ & $4(13.8)$ & $<0.01^{a}$ \\
\hline $1-2$ & $75(61.5)$ & $55(59.1)$ & $20(69.0)$ & \\
\hline$>2$ & $8(6.5)$ & $3(3.2)$ & $5(17.2)$ & \\
\hline
\end{tabular}

Abbreviations: AKI, acute kidney injury; ICU, intensive care unit; IQ, interquartile range; APACHE II, Acute Physiology and Chronic Health Evaluation II; ICU, intensive care unit. ${ }^{a} \chi^{2}$ de Pearson; bMann-Whitney; 'Length 
of hospital stay prior to ICU admission was not included in this analysis.

2 
Table 2 (on next page)

Drug classification according to the first level of ATC 
1

Table 2. Drug classification according to the first level of ATC.

Anatomical groups

Frequency, n (\%)

$\mathrm{N}:$ Nervous system

$658(24.3)$

A: Alimentary tract and metabolism

$569(21.0)$

B: Blood and blood forming organs

$487(18.0)$

C: Cardiovascular system

$396(14.6)$

$\mathrm{J}$ : Antiinfectives for systemic use

$313(11.6)$

m: Musculo-skeletal system

$84(3.1)$

R: Respiratory system

$80(3.0)$

$\mathrm{H}$ : Systemic hormonal preparations, excl. sex hormones and

$56(2.1)$ insulins

D: Dermatologicals

$26(1.0)$

$\mathrm{V}$ : Various

$23(0.9)$

S: Sensory organs

$10(0.4)$

P: Antiparasitic products, insecticides and repellents

$2(0.1)$

Abbreviation: ATC, Anatomical Therapeutic Chemical Classification. 
Table 3 (on next page)

Frequency of potentially nephrotoxic drugs 
Table 3. Frequency of use of potentially nephrotoxic drugs in the ICU patients studied at a teaching hospital $(n=122)$.

\begin{tabular}{|c|c|c|c|}
\hline Potentially nephrotoxic drugs & $\begin{array}{c}\text { Frequency }^{*}, \mathrm{n} \\
(\%)\end{array}$ & Potentially nephrotoxic drugs & $\begin{array}{c}\text { Frequency }^{*}, \\
\text { n (\%) }\end{array}$ \\
\hline Fentanyl & $86(70.5)$ & Propofol & $15(12.3)$ \\
\hline Morphine & $79(64.8)$ & Dobutamine & $14(11,5)$ \\
\hline Omeprazole & $78(63.9)$ & Amikacin & $13(10.7)$ \\
\hline Norepinephrine & $73(59.8)$ & Amiodarone & $12(9.8)$ \\
\hline Magnesium sulfate & $54(44.3)$ & Carvedilol & $12(9.8)$ \\
\hline Furosemide & $46(37.7)$ & Spironolactone & $12(9.8)$ \\
\hline Vancomycin & $37(30.3)$ & Amoxicillin + clavulanic acid & $11(9.0)$ \\
\hline Diazepam & $35(28.7)$ & Ciprofloxacin & $11(9.0)$ \\
\hline Simvastatin & $33(27.1)$ & Valproic acid & $10(8.2)$ \\
\hline Metronidazole & $32(26.2)$ & Clopidogrel & $10(8.2)$ \\
\hline Ceftriaxone & $31(25.4)$ & Non-ionic contrast media & $10(8.2)$ \\
\hline Meropenem & $31(25.4)$ & Hydralazine & $10(8.2)$ \\
\hline Polymyxin & $31(25.4)$ & Clarithromycin & $9(7.4)$ \\
\hline Clonazepam & $27(22.1)$ & Clonidine & $9(7.4)$ \\
\hline Phenytoin & $27(22,1)$ & Gentamicin & $9(7.4)$ \\
\hline Captopril & $25(20.5)$ & Calcium gluconate & $9(7.4)$ \\
\hline Amlodipine & $24(19.7)$ & Phenobarbital & $8(6.6)$ \\
\hline Risperidone & $19(15.6)$ & Clindamycin & $7(5.8)$ \\
\hline Losartan & $17(13.9)$ & Epinephrine & $7(5.8)$ \\
\hline Sodium nitroprusside & $17(13.9)$ & Hydrochlorotiazide & $7(5.7)$ \\
\hline Acetaminophen & $17(13.9)$ & Oxacillin & $7(5.7)$ \\
\hline Piperacillin + tazobactam & $17(13.9)$ & Atropine & $6(4.9)$ \\
\hline Tramadol & $17(13.9)$ & Cefepime & $6(4.9)$ \\
\hline Cefazolin & $15(12.3)$ & & \\
\hline
\end{tabular}

${ }^{*}$ Potentially nephrotoxic drugs with frequency of use $<5$ patients were not shown in this table.

${ }^{* *}$ Iohexol or iopamidol or ioversol or iobitriol 


\section{Table 4(on next page)}

Final model of logistic regression 
1

Table 4. Final model of logistic regression for the factors associated with acute kidney injury in intensive care unit patients of a teaching hospital.

\begin{tabular}{|c|c|c|}
\hline Variables & Odds Ratio & $95 \% \mathrm{Cl}$ \\
\hline Age & 1.02 & $0.99-1.05$ \\
\hline \multicolumn{3}{|l|}{ Race } \\
\hline Non-white & 1.00 & - \\
\hline White & 1.28 & $0.40-4.10$ \\
\hline \multicolumn{3}{|l|}{ Comorbidities } \\
\hline 0 & 1.00 & - \\
\hline $1-2$ & 1.29 & $0.34-4.93$ \\
\hline$>2$ & 0.95 & $0.20-4.45$ \\
\hline \multicolumn{3}{|l|}{ Length of ICU staya (days) } \\
\hline Up to 5 days & 1.00 & \\
\hline From 6 to 15 & 1.64 & $0.42-6.39$ \\
\hline More than 15 & 0.24 & $0.28-2.02$ \\
\hline Number of drugs in general & 1.15 & $1.05-1.27$ \\
\hline $\mathrm{S}_{\mathrm{Cr}}$ at hospital admission & 2.83 & $0.25-31.60$ \\
\hline GFR at hospital admission & 1.00 & $0.98-1.01$ \\
\hline APACHE $\|$ at admission ICU & 0.99 & $0.93-1.06$ \\
\hline \multicolumn{3}{|c|}{$\begin{array}{l}\text { Abbreviations: AKI, acute kidney injury; ICU, intensive care unit; Cl, } \\
\text { confidence interval; } \mathrm{S}_{\mathrm{Cr}} \text {, serum creatinine; GFR, glomerular filtration rate; } \\
\text { APACHE II, Acute Physiology and Chronic Health Evaluation II (APACHE II). } \\
\text { aLength of hospital stay prior to ICU admission was not included in this }\end{array}$} \\
\hline
\end{tabular}




\section{Figure 1 (on next page)}

Flowchart for patient selection and follow-up until ICU discharge

Abbreviations: AKI, acute kidney injury. 
Patients assessed for eligibility $(n=296)$

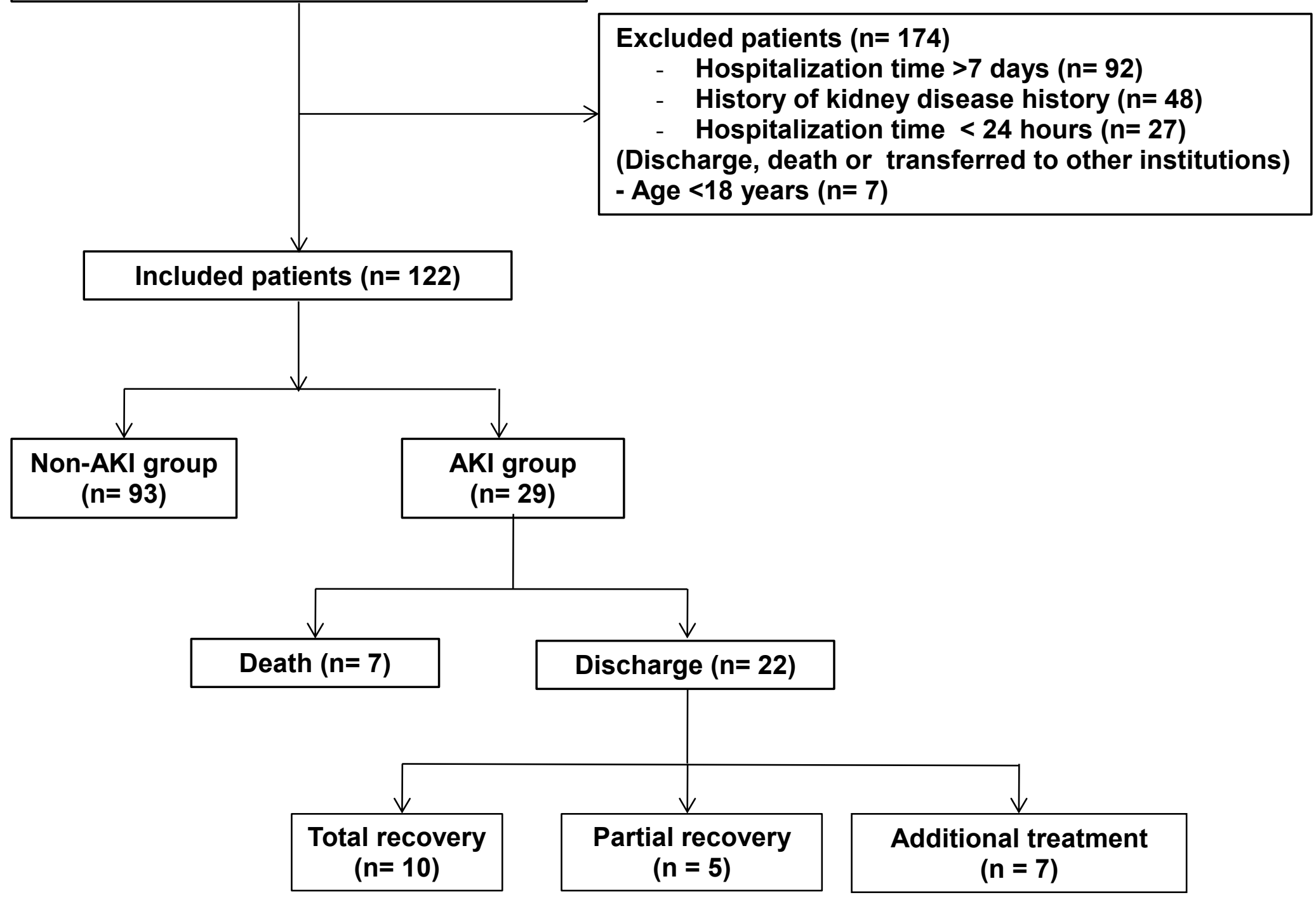


Figure 2

$\mathrm{S}_{\mathrm{Cr}}(\mathrm{A})$ and GFR (B) at three different moments of hospitalization according to AKI and non-AKI groups

Abbreviations: $S_{C r}$, serum creatinine; $A K I$, acute kidney injury; GFR, glomerular filtration rate.
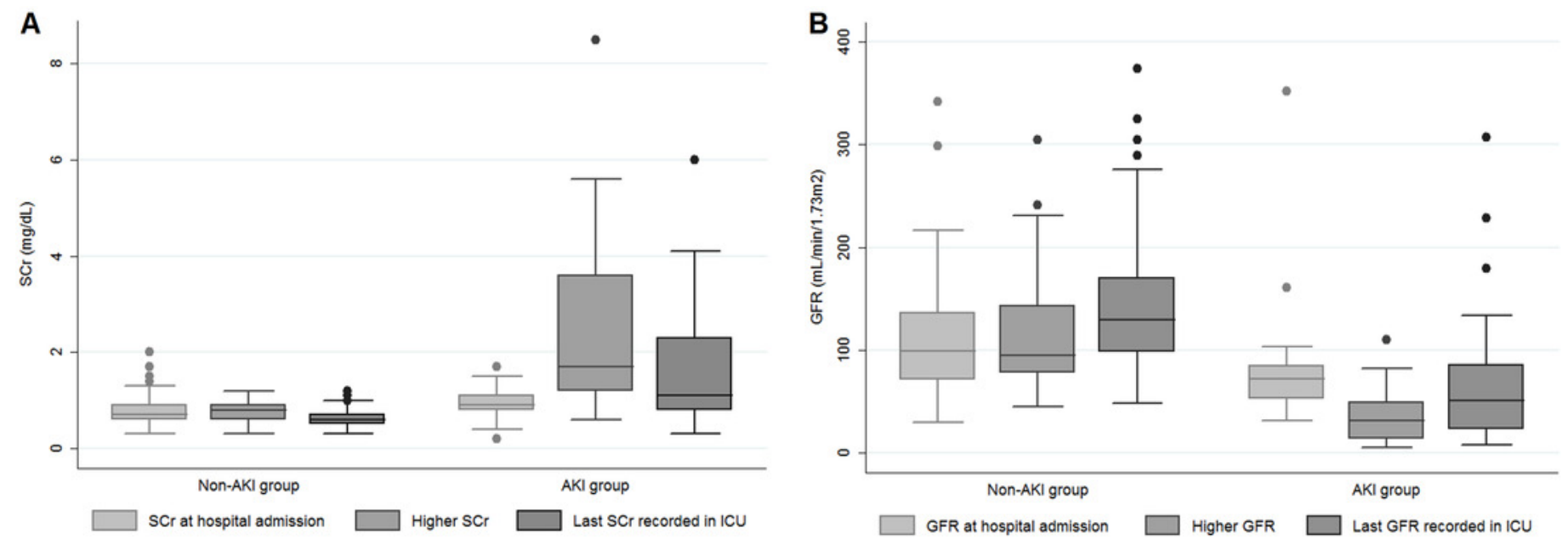
Figure 3

ROC curve for the final model

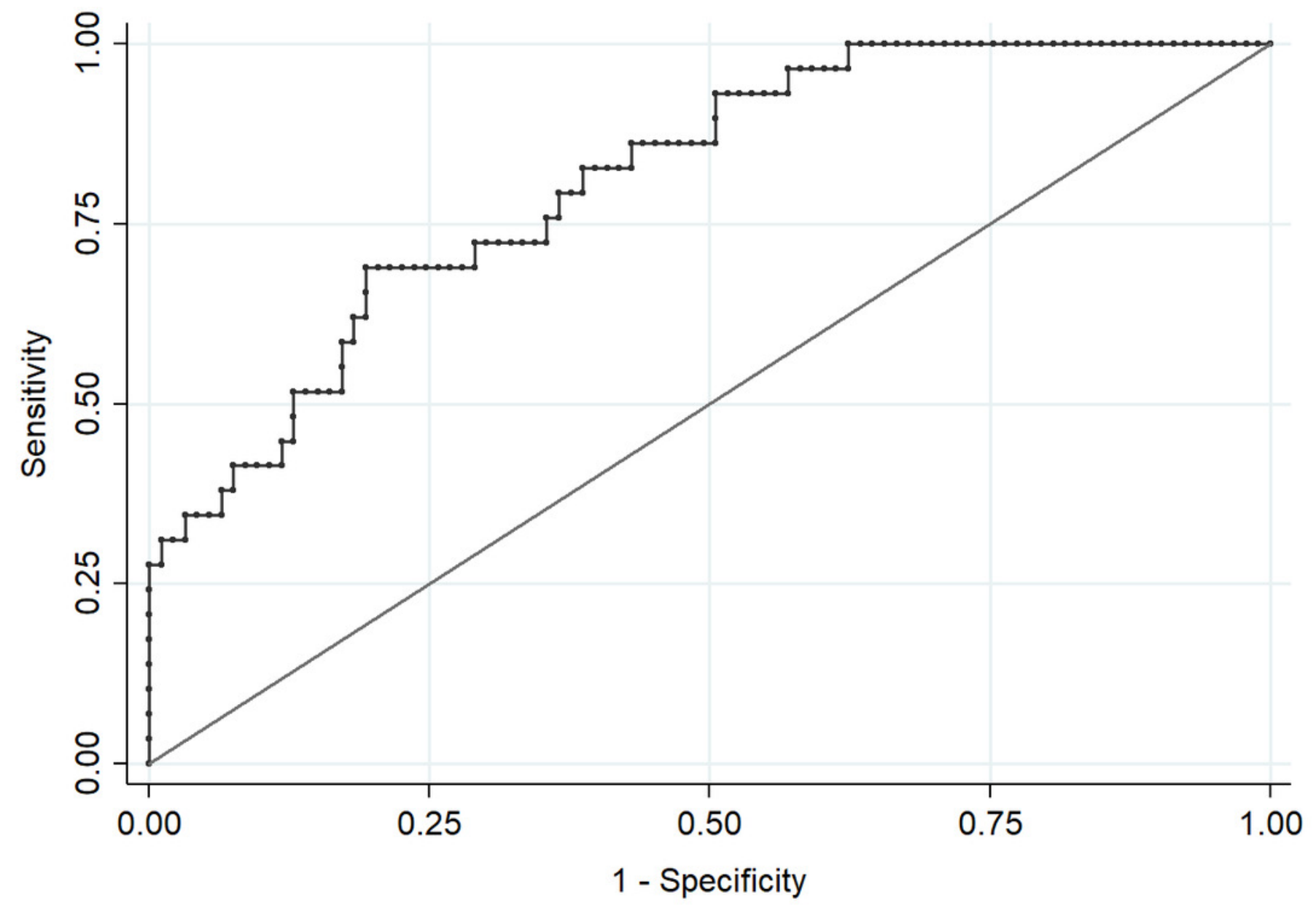

Area under ROC curve $=0.8102$ 
Figure 4

Probability of occurrence of AKI according to the number of drugs used by the studied ICU patients

Abbreviations: AKI, acute kidney injury; ICU, intensive care unit.

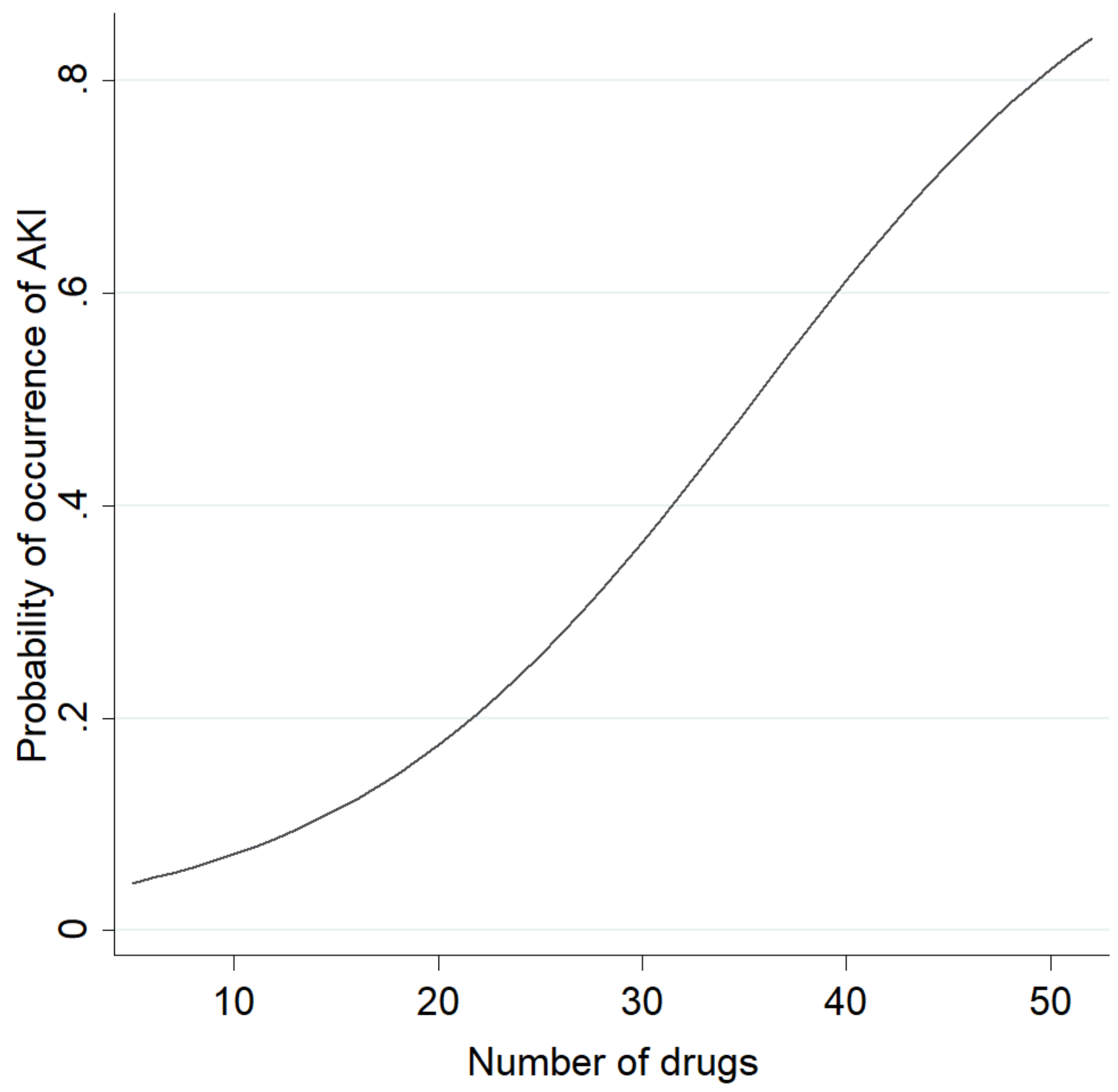

